(Aus dem pnysiologischen Institut der Universität Königsberg i. Pr.)

\title{
Versuche über die Wirkung von Entladungsschlägen auf Blut und auf halbdurchlässige Membranen. \\ Von
}

\section{Hermann.}

(Mit 9 Textfiguren.)

\section{Versuche mit Blut.}

In einer früheren Arbeit ${ }^{1}$ ) habe ich gezeigt, dass (lie Wirkung hochgespannter Induktionsströme auf die Blutkörper ungemein ähnlich ist derjenigen der Erwärmung, und da die Durchleitung solcher Ströme durch eine dünne Blutschicht mit sehr erheblicher, leicht fühlbarer und durch Paraffinstückchen nachweisbarer Erwärmung verbunden ist, die Vermuthung ausgesprochen, dass die von Rollett entdeckte Aufhellung des Blutes durch elektrische Einwirkungen lediglich Wirkung der Erwärmung sei.

Rollett hat bald darauf in einer sehr ausführlichen Abhandlung ${ }^{2}$ ) zwar zugegeben, dass die Aufhellung, wenn sie durch Induktionsströme bewirkt wird, auf Erwärmung beruht, aber Gründe und weitere Versuche dafür beigebracht, dass diese Auffassung niclit ausreicht, die Wirkung von Entladungsschlägen zu erklären. Ich hatte bis dahin die Aufhellung immer nur, nach E. Neumann's Vorgang, mit Induktionsströmen hervorgebracht, und wandte mich nun erst, nach Erscheinen der Rollett' schen Arbeit, zu Versuchen mit Entladungsschlägen.

Anfangs verwandte ich hierzu die Entladungen grosser Leidener Flaschen, einzeln oder in Batteriezusammenstellung, später, da die Anschaffung eines grösseren Kondensators zu anderen Zwecken nöthig

1) Dieses Arehiv Bd. 74 S. 164. 1899.

2) Dieses Archiv Bd. 82 S. 199. 1900. 
wurde, diejenigen eines Hochspannungs-Kondensators von Edelmann von ähnlicher Einrichtung wie der von Rollett benutzte. Derselbe besteht aus 5 Abtheilungen (mit Glasplatten), welche einzeln und kombinirt bersutzt werden können; im letzteren Falle beträgt die Kapazität 0,0247 Mikrofarad. Die Anordnung zur Ladung und Entladung war ganz analog der von Rollett (a. a. O.) beschriebenen. Die ladenden Induktionsströme, in deren Kreis eine Funkenstrecke von einigen Centimetern (Platte und Spitze) eingeschaltet war, wurden von einem Ruhmkorff'schen Induktor von $20 \mathrm{~cm}$ Funkenlänge geliefert. Der primäre Kreis enthielt als Kette zwei neben einander geschaltete Akkumulatorketten von je 15 Elementen mit Vorschaltwiderstand, und einen Quecksilberunterbrecher, welcher durch eine besondere Kette getrieben wurde. Diese Angaben mache ich nur wegen der in der zweiten Abtheilung beschriebenen neuen Versuche.

Zunächst wiederholte ich Rollett's Trogversuche, zu welchen ich eine Anzahl sehr geeigneter, im Institut vorräthiger Tröge verwandte, welche ganz aus Glasplatten zusammengesetzt sind. Sie haben alle im Lichten $25 \mathrm{~mm}$ Höhe, $30 \mathrm{~mm}$ Breite und Dicken von 2,5 bis gegen $10 \mathrm{~mm}$. Als Elektroden verwandte ich Zinkblechstreifen von der Form $a \prod b$, deren Ende $a$ die eine Schmalseite des Innenraums bedeckt, während das Ende $b$ eine Reitklemme für den Leitungsdraht trägt.

Die Angabe Rollett's, dass die Aufhellung durch Entladungsschläge ohne erhebliche Erwärmung des Blutes eintritt, bestätigte sich sofort, am schönsten bei der von Rollett angegebenen Verwendung eines Gemisches von Blut und 10 procentiger Kochsalzgelatine. Das erstarrte Gemisch hellt sich durch eine mässige Zahl von Entladungsschlägen auf, obne zu schmelzen, während es schon durch die Wärme der Hand geschmolzen wird.

Um auch mit dem Finger (analog den Versuchen mit Induktionsströmen unter dem Deckglas) den Grad der Erwärmung verfolgen zu könuen, verfertigte ich mir aus Glasplatten mittels Kanadabalsams einen kleinen Trog, dessen Vorderwand nur aus einem grossen, dünnen Deckglase bestand. Hier konnte ich mich auch mit dem Finger überzeugen, dass eine Blutschicht durch Entladungsschläge aufgehellt werden kann, ohne dass fühlbare Erwärmung stattfindet.

Leider lässt sich die Wirkung sehr kräftiger Entladungen auf Blutschichten von kapillarer Dünne, wie sie für die mikroskopische Beobachtung nöthig ist, nicht direkt beobachten, weil statt Durch- 
strömung der dünnen Schicht stets Funken zur Seite derselben zwischen den Elektroden oder zwischen letzteren und dem Metall des Objektivs überspringen. Ja sogar wenn man das Präparat durch sehr lange feuchte Fliesspapierstreifen (ich ging bis $14 \mathrm{~cm}$ Länge derselben) von den Stanniolelektroden trennt, zieht die Elektrizität es vor, zwischen den beiden Papierstreifen neben dem Deckglase in riesigen Funken überzuspringen, statt durch die dünne Blutschicht sich abzugleichen. Man muss, um letzteres zu erreichen, die Intensität des Vorganges sehr bedeutend abschwächen. Auch bei Trogversuchen kann dies Ueberspringen von Funken über die Oberfläche der Flüssigkeit hinweg sich einstellen. Ich habe hierbei sehr oft die Erfahrung gemacht, welche vermuthlich den Experimentatoren auf diesem physikalischen Gebiet nicht neu ist, obwohl ich sie nirgends erwähnt finde, dass unmittelbar neben einer schlechtleitenden Flüssigkeitsschicht weit längere Funken überspringen, als die eigentliche Funkenstrecke giebt.

Zur vollständigeren Aufklärung über die Beziehungen zwischen der erwärmenden und der blutkörperzerstörenden Wirkung der Elektrizität und die Bedeutung des Kondensators für die Wirkungen habe ich systematische Versuchsreihen ohne und mit Kondensator, mit und ohne Funkenstrecken, und bei allen möglichen Variationen der Anordnung angestellt.

Bei diesen Versuchen wurde, um sie nicht allzusehr auszudehnen, stets der gleiche Trog (im Lichten $30 \mathrm{~mm}$ lang, 25 hoch, $5^{1 / 4}$ dick) und bei stets gleicher Flüssigkeitshöhe verwendet, ferner stets das angegebene Induktorium und der Kondensator bei voller Kapazität. Als Flüssigkeiten dienten: Leitungswasser, 0,6 procentige Kochsalzlösung und defibrinirtes Rindsblut. In den Trog tauchte stets ein Thermometer mit sehr kleinem Gefäss.

Fig. 1 zeigt schematisch alle verwendeten Anordnungen; $R$ ist die sekundäre Spirale (20 cm Funkenlänge), $K$ der Kondensator (1/40 Mikrofarad), $S$ der Absperrschlüssel für den Trog T. $a$ und $b$ sind Funkenstrecken, $a$ zwischen Platte und Spitze, $b$ zwischen zwei abgerundeten Zinkstäben von $5 \mathrm{~mm}$ Dicke (Kohl'sches Funkenmikrometer).

Die Funkenstrecke $a$ kann innerhalb der Leistungsfähigkeit des Induktors beliebig gross sein, bis $20 \mathrm{~cm}$, obwohl für jede Anordnung ein Optimum derselben existirt. Die in den Anordnungen 7 und 8 (die letztere ist die von Rollett benutzte) angebrachte Funken- 
strecke $b$ darf bei Anordnung 7 nur wenige (bis etwa 4) Millimeter lang sein; bei grösserer Länge springen keine Funken über. Bei Anordnung 8 dagegen kann, weil der Kondensator nicht dureh die Spirale geschlossen ist, die Funkenstrecke $b$ bis über $20 \mathrm{~mm}$ gross gemacht werden. Natürlich sind die Funken in $b$ um so heftiger und seltener, je länger die Strecke. Stets aber, und ebenso bei Anordnung 7, haben dieselben den Charakter der Entladungsfunken, sie sind weiss und knallend, während diejenigen der Strecke $b$ sprühend und bläulich sind. Die Einschaltung des Trogwiderstandes in die
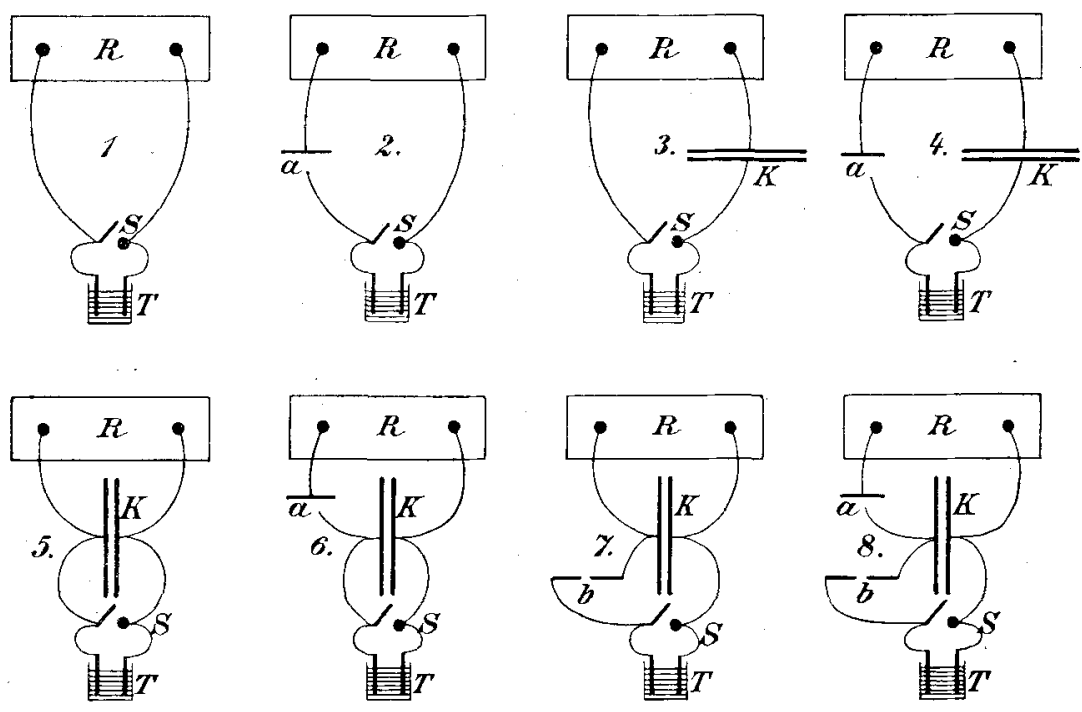

Fig. 1.

Leitung $K T b K$, d. h. die Oeffnung des Schlüssels $S$, macht, wie bekannt, die Funken erheblich schwächer, hat jedoch auf die zulässige Länge der Funkenstrecke keinen Einfluss.

Die folgende Uebersicht zeigt nun zunächst den Effekt der Anordnungen auf die drei verwendeten Flüssigkeiten. Die angegebenen Grade bedeuten die Temperaturzunahme der Flüssigkeit in 1 Minute; die Anfangstemperatur war stets dieselbe. 


\begin{tabular}{|c|c|c|c|c|}
\hline $\begin{array}{c}\text { An- } \\
\text { ordnung }\end{array}$ & $\begin{array}{l}\text { Leitungs- } \\
\text { wasser }\end{array}$ & $\begin{array}{l}0,6 \text { proc. } \\
\text { Kochsalz- } \\
\text { lösung }\end{array}$ & Blut & Bemerkungen \\
\hline $\begin{array}{l}1 . \\
2 . \\
3 . \\
4 . \\
5 . \\
6 . \\
7 . \\
7 . \\
8 . \\
8 . \\
8 . \\
8 . \\
8 . \\
8 . \\
8 . \\
8 . \\
8 .\end{array}$ & $\begin{array}{c}20-27^{0} \\
\left.7-16^{0 *}\right) \\
16-27^{0} \\
\left.3,2-16^{0 *}\right) \\
28,5^{0} \\
8-26,5^{0} \\
30-43,5^{0} \\
50-53^{0} \\
34^{0} \\
37^{0} \\
27^{0} \\
86^{0} \\
13^{0} \\
28-28^{\circ} \\
18-22,5^{0} \\
\overline{7^{0}}\end{array}$ & $\begin{array}{c}\left\langle 0,5^{\circ}\right. \\
0 \\
0 \\
0 \\
0,5^{0} \\
0,3^{0} \\
29-40^{0} \\
46,5-49,5^{0} \\
-- \\
- \\
- \\
- \\
- \\
19-230 \\
- \\
6,5^{0}\end{array}$ & $\begin{array}{c}1,2^{0} \\
0 \\
0,3^{\circ} \\
0 \\
1-2^{0} \\
0 \\
30-42^{\circ} \\
58,5-60^{\circ} \\
- \\
- \\
- \\
- \\
17^{\circ} \\
20-22^{\circ} \\
26-28^{\circ} \\
15^{\circ} \\
7^{\circ}\end{array}$ & $\begin{array}{l}\text { *) Um so höher, je kürzer } \\
\text { die Strecke } a \text {. } \\
\text { *) Um so höher, je kürzer } \\
\text { die Strecke } a \text {. } \\
\text { Strecke } b=1-2 \mathrm{~mm} \\
\text { Strecke } b=3-4 " \\
a=2, b=2 \mathrm{~mm} \\
a=3, b=3 " \\
a=5, b=1 " \\
a=5, b=2 " \\
a=30, b=1 \\
a=30, b=3-4 \mathrm{~mm} \\
a=30, b=9 \mathrm{~mm} \\
a=30, b=20 " \\
a=80, b=9 "\end{array}$ \\
\hline
\end{tabular}

Die Versuche ergeben hiernach Folgendes:

1. Bei den Anordnungen $1-6$, bei welchen der Kondensator nicht zu wesentlichem Einfluss gelangen kann, rühren die thermischen Effekte wesentlich von den Induktionsströmen direkt her. Die Erwärmung ist beim Leitungswasser durchweg am grössten, beim Blute und noch mehr bei der Kochsalzlösung sehr unbedeutend. Dies ist leicht begreiflich; denn der Widerstand der Trogflüssigkeit ist, selbst bei Leitungswasser, entweder kleiner oder doch nicht wesentlich grösser als derjenige der Spirale ${ }^{1}$ ). Nennen wir ersteren $w$, denjenigen des übrigen Kreises, also wesentlich den der Spirale, $W$, so ist die Erwärmung im Troge proportional der Grösse

$$
\frac{E^{2} w}{(W+w)^{2}}
$$

und diese Grösse nimmt mit $w \mathrm{zu}$, so lange nicht $w>W$.

2. Ist, wie in den Anordnungen 2, 4 und 6, eine Funkenstrecke $a$ im Kreise, so setzt dies die Erwärmungen wesentlich herab. Dies rührt grösstentheils davon her, dass die Strecke $a$ nur die Oeffnungs-Induktionsströme zu Stande kommen lässt, wodurch

1) Letzterer ist etwa $13300 \mathrm{Ohm}$, derjenige des Troges bei 0,6 procentiger Salzlösung etwa $300 \mathrm{Ohm}$. Bei meinen früheren Versuchen mit kapillarer Blutschicht (dieses Archiv Bd. 74 S. 164) war natürlich die Erhitzung enorm viel bedeutender, und ferner um so grösser, je besser das Leitungsvermögen, wie a. a. $O$. ausgeführt ist. 
dem Troge ziemlich die Hälfte der Energie verloren geht. Ausserdem nimmt aber die Funkenstrecke selbst einen Theil der Energie in Anspruch.

3. Bei der Anordnung 7 tritt bei Weitem die beträchtlichste Erhitzung im Troge ein, so dass derselbe schon nach ganz kurzer Zeit trotz seiner über $4 \mathrm{~mm}$ dicken Glaswände sich von aussen heiss anfühlt. Ferner fällt sofort auf, dass hier die Erhitzung nicht vom Widerstande abhängt, sondern sogar beim Blute grösser sein kann als bei dem Leitungswasser. Die Erhitzung nimmt mit der Länge der Funkenstrecke in $b$ dentlich $\mathrm{zu}$, obwohl dieselbe nicht über 3-4 mm getrieben werden kann (s. oben). Das Blut wird bei Anordnung 7 sehr leicht aufgehellt, aber stets erst bei Temperaturen von etwa $58^{\circ}$. Sobald die Durchleitung etwas länger dauert, tritt Hämoglobinzersetzung und Eiweissgerinnung ein. Bemerkt sei noch, dass unter sonst gleichen Umständen die Erwärmung mit der Kapazität des Kondensators zunimmt.

4. Bei Anordnung 8 (der Rollett'schen) ist die Erhitzung sehr viel geringer als bei Anordnung 7, aber auch hier, ganz abweichend von den Versuchen mit den Anordnungen $1-6$, vom Leitungsvermögen kaum abhängig und oft bei Blut grösser als bei Wasser. Die Erwärmung ist, wie bei Anordnung 2, 4 und 6, um so geringer, je länger die Funkenstrecke; dagegen wirkt, wenn man die Strecke $a$ konstant hält (z. B. $=30 \mathrm{~mm}$ ), die Verlängerung der Funkenstrecke $b$, gerade wie bei Anordnung 7 , erhöhend auf die Erwärmung, aber nur bis zu einem gewissen Optimum; weitere Verlängerung setzt die Erwärmung wieder herab.

Während aber die Erwärmung bei dieser Anordnung durchweg geringer ist als bei Anordnung 7, erfolgt die Aufbellung des Blutes hier, sobald die Funkenstrecke $b$ einigermassen beträchtlich ist, ungemein leicht, und oft schon bei.Temperaturerhöhungen von nur $6-7^{0}$.

Um nun zunächst die physikalischen Erscheinungen bei den Anordnungen 7 und $8 \mathrm{zu}$ verstehen, muss man die Rolle des Kondensators in allen Versuchen etwas näher betrachten. Hierbei muss festgehalten werden, dass die von dem Induktor gelieferten Ströme zwar nicht ohne Weiteres mit wirklichen Wechselströmen identifizirt werden dürfen, weil bekanntlich die Oeffnungsströme einen ganz anderen zeitlichen Verlauf haben als die Schliessungsströme, dass sie aber für die Ladung des Kondensators sich doch im Wesent- 
lichen wie wahre sinusoidale Wechselströme verhalten müssen,' weil die Ladung von der Elektrizitätsmenge $\int_{0}^{\infty} i d t$ abhängt, und diese für die Schliessungs- und Oeffnungsströme dieselbe ist. Nur bei sehr schnellem Spiel des Unterbrechers, wie es aber in unserm Falle nicht vorhanden, würden die beiden mit einander abwechselnden entgegengesetzten Ladungen ungleich sein. Im Allgemeinen können also die bekannten Gesetze der Wechselströme in Kreisen, welche Kapazität und Selbstinduktion besitzen, auf unsern Fall Anwendung finden. Dass mit Funkenstrecke $a$ nur die Oeffnungsströme in Betracht kommen, ist schon erwähnt. Es bedarf also auch keiner weiteren Erklärung, dass die Erwärmungen in den Anordnungen mit grader Zahl geringer sind als in den ihnen entsprechenden mit ungrader.

Bei Anordnung 3 kann der Kondensator keine anderen Wirkungen entfalten als diejenigen, welche er nach bekannter Theorie in jedem Wechselstromkreis hat; $d$. h. er kompensirt mehr oder weniger die phasenverschiebenden und intensitätsschwächenden Wirkungen der hohen Selbstinduktion der sekundären Spirale. In Folge dessen müssen die Erwärmungen hier etwas grösser ausfallen als bei Anordnung 1; dass hiervon in den Versuchen nichts zu erkennen ist, liegt nur daran, dass das Messverfahren den Umständen gemäss sehr primitiv ist.

Bei Anordnung 5 liegt der Kondensator im Nebenschluss zwischen Spirale und Trogleitung. Für diesen Fall ergiebt die Theorie ${ }^{1}$ ), dass er ebenfalls bis zu einer gewissen Höhe der Kapazität auf den Strom in der Trogleitung verstärkend, bei höheren Kapazitäten schwächend wirken muss. Auch hier kann seine Wirkung nicht erheblich sein, und in der That zeigt sich kein wesentlicher Einfluss bei Vergleichung der Erwärmung mit derjenigen der Anordnung 1.

Ganz anders wirkt der Kondensator bei Anordnung 7. Er erreicht hier höhere Potentialdifferenzen als bei Anordnung 5 , wo er beständig einen Nebenschluss durch die Trogleitung hat. So oft nun die Potentialdifferenz gross genug wird, um die Funkenstreeke $b$ (welche hier nur gering sein darf) zu überwinden, findet eine Entladung statt, und obwobl dieselbe zwei Wege hat, nämlich durch

1) Da in den Hand- und Lehrbüchern dieser Fall nicht behandelt wird, habe ich die theoretische Untersuchung desselben selbst durehgeführt, unterlasse es aber, dieselbe hier darzustellen. 
die Spirale und durch die Funkenstrecke mit oder ohne den Trog, wählt sie fast ausschliesslich den letzteren Weg, weil gegenüber den raschen Oszillationen der Entladung der scheinbare Widerstand der Spirale wegen ihrer hohen Selbstinduktion enorm vergrössert wird ${ }^{1}$ ). Dies ist Jie Ursache der starken Erhitzung bei dieser Anordnung. Auch ist leicht zu erklären, warum hier Salzlösung und Blut ebenso stark oder selbst stärker erwärmt werden als Wasser. Je geringer nämlich der Gesammtwiderstand der Trogleitung, um so mehr Entladungsstrom lenkt sie von der Spirale auf sich $a b$, und hierdurch wird die bei gleicher Stromstärke höhere Erwärmung der schlechter leitenden Flüssigkeit kompensirt oder überkompensirt. Durch Anordnung 7 werden also mittels des Kondensators der Trogflüssigkeit weit grössere Energiemengen zugeführt als ohne denselben (Anordnung 2).

Bei Anordnung 8 (der von Rollett benutzten) nimmt der Kondensator, wegen der nur einsinnigen Ladung und der Trennung von der Spirale die höchsten Ladungen an, und die Entladung kann selbst lange Funkenstrecken überwinden. Auch hier muss die Erwärmung aus dem angegebenen Grunde (weil der Trogwiderstand der wesentliche der Leitung ist) vom spezifischen Leitungsvermögen wenig abhängig sein. Sie ist aber hier sehr viel geringer als bei Anordnung 7, weil die Gesammtenergie nur diejenige der Oeffnungsinduktionen ist; ganz abgesehen von Zerstreuungen durch Unvollkommenheiten des Kondensators.

Die auffallende Erscheinung, dass die Trogflüssigkeit bei Anordnung 7 so sehr viel mehr Energie empfängt als z. B. bei Anordnung 1, ist natürlich kein Widerspruch gegen das Energiegesetz; denn erstens wird sicher, wenn die Trogflüssigkeit stärker erwärınt wird, der Draht der Spirale weniger erwärmt; zweitens aber entwickelt der ganze sekundäre Kreis bei Anordnung 7 weit mehr Energie als bei Anordnung 1, was sich am primären Strome nachweisen lässt, welcher die Energie der sekundären Ströme hergeben muss. Ich habe stets im primären Kreise ein Ampèremeter, welches die mittlere Stromstärke anzeigt, und es zeigt sich regelmässig bei

1) Bekanntlich springen durch eine Funkenstrecke, durch welche man einen geladenen Kondensator entlädt, auch dann noch Funken über, wenn dieselbe eine sehr gut leitende Nebenschliessung hat. Der Grund ist auch hier die Selbstinduktion der letzteren, selbst wenn dieselbe keine Windungen enthält. 
Anordnung 7 eine erheblich kleinere Ablenkung desselben als bei allen übrigen Anordnungen.

Die Versuche mit Anordnung 7 ergänzen zunächst die Rollett'schen dahin, dass man auch in grösseren Trögen unter gewissen Umständen, ganz wie bei Neumann's und meinen Versuchen mit kapillaren Blutschichten, erst dann Aufhellung des Blutes erhält, wenn die Temperatur auf solche Höhen gestiegen ist, welche auch ohne Elektrizität die Blutkörper auflösen. Dagegen bestätigen die Versuche mit Anordnung 8 Rollett's Angabe, dass es ausserdem eine von der Temperaturerhöhung unabhängige rein elektrische Aufhellung des Blutes giebt.

Es entsteht nun weiter die Frage, ob diese rein elektrische Aufhellung auch bei anderen Einwirkungen als Anordnung 8, wenn auch träger, zu Stande kommen kann, mit anderen Worten, ob der Vorgang der Funkenentladung des Kondensators für diese Wirkung unumgänglich nothwendig oder nur der günstigste ist. Mit Anordnung 7 lässt sich diese Frage nicht entscheiden, einmal weil auch hier Kondensatorentladungen mitspielen, zweitens weil unsere Frage sich nur dadurch beantworten liesse, dass die Aufhellung schon etwas vor Erreichung der an sich Aufhellung bewirkenden Temperatur auftreten kann, wovon ich jedenfalls nichts Sicheres feststellen konnte.

Ich habe daher zunächst die gewöhnlichen Wechselströme eines Schlitteninduktoriums volle 24 Stunden lang durch den Bluttrog gehen lassen; die Temperatur stieg hier nie mebr als $1^{0}$ über die Zimmertemperatur. Es trat keine Aufhellung ein; oben bildete sich durch Senkung eine Serumschicht.

Ferner wurden die Ströme des grossen Induktors von $20 \mathrm{~cm}$ Funkenlänge 9 Stunden lang (Anordnung 1) durch den Trog geleitet. Die Temperatur des Blutes hielt sich während dieser Zeit auf $27-29^{\circ}$, bei etwa $14^{\circ}$ Zimmertemperatur. Auch hier trat zwar erhebliche Verdunkelung, aber durchaus keine Lackfarbe ein ${ }^{1}$ ); neben Senkung der Blutkörper zeigte sich oben etwas feinblasiger Schaum durch Gasentwicklung von den Elektroden. Nach Schluss des Versuches bewirkte Anordnurg 8 in wenigen Sekunden vollständige Aufhellung.

1) Ausser dem direkten Anblick wurden auch fortlaufend mikroskopische Proben zur Feststellung der Unversehrtheit der Blutkörper entnommen. 
Es ist hierdurch festgestellt, dass in der That für die rein elektrische Aufhellung Kondensatorentladungen prinzipielle Bedingung sind.

Schliesslich habe ich mich noch bemüht, festzustellen, wie schnell günstigsten Falies die Blutkörper entfärbt werden können. Alle meine Bemühungen, die Wirkung stärkster Entladungen direkt unter dem Mikroskop zu verfolgen, misslangen. Auf die mannigfaltigste Art versuchte ich, den Entladungsvorgang auf eine kapillare Blutschicht unter dem Deckglase wirken zu lassen. Entweder springen, wie schon bemerkt, statt des Durchganges riesige Funken neben dem Deckglase oder über dasselbe hinweg zwischen den Stanniolplatten über, oder das Deckglas wird zertrümmert und weggeschleudert $^{1}$ ). Dies ist namentlich der Fall, wenn man den Kreis weit offen lässt, so dass der Kondensator sich bis zu seiner vollen Kapazität laden kann, und dann mit dem Entlader eine Entladung bewirkt. Es ist schlechterdings unmöglich, eine so grosse Elektrizitätsmenge auf einmal durch eine kapillare Blutschicht, sei sie kurz oder lang, gehen zu lassen, ohne dass die Flüssigkeit unter plötzlicher Dampfbildung zersprengt wird.

Durch allmähliche Annäherung an die Grenze der Elektrizitätsmenge, welche die Flüssigkeit noch wirklich zu leiten vermag, gelang es endlich doch zum Ziele zu kommen. Hierzu musste ein Zwischenraum von etwa $50 \mathrm{~mm}$ zwischen den Stanniolrändern mit einer Blutschicht bedeckt, und ein Objektträger von gewöhnlichem Format so aufgelegt werden, dass unter ihm eine gleichmässig dicke, immerhin noch ganz dünne Blutschicht sich befand. Der Objektträger gestattete noch die Anwendung des Objektives $B B$. Ferner musste die Kapazität des Kondensators meist auf $3 / 5$ ihrer vollen Grösse reduzirt werden. Bei der Entladung mittels des Entladers entstand jetzt nur ein schwacher Funke. Fig. 2 zeigt schematisch die Anordnung des Versuchs. $O$ ist der Objektträger, $E$ der Entlader. Hier nun genügte eine einzige Entladung, um die Blutschicht zwar nicht im Augenblick, aber in einigen Sekunden, vollkommen lackfarbig zu machen. Die vollständige Entfärbung der Blutkörper wurde stets

1) Zu diesen und den folgenden Versuchen dienten sehr grosse (für Stativ I Zeiss passende) Objektträger von $190 \mathrm{~mm}$ Länge und $76 \mathrm{~mm}$ Breite. Von jedem Ende geht ein $30 \mathrm{~mm}$ breiter Stanniolstreifen longitudinal bis zu einem freien Felde von 50 mm Länge. Auf beiden Enden sitzen Reitklemmen. 
nachher durch Auflegen eines gewöhnlichen Deckglases bei stärkerer Vergrösserung festgestellt; es zeigten sich nur noch Stromata. Sehr charakteristisch zeigt sich bei solchen Versuchen, wenn unter dem Objektträger, wie es sehr leicht geschieht, einige plattgedrückte Luftblasen sich befinden, auf beiden. Seiten derselben in longitudinaler Richtung eine kurze opak gebliebene Strecke, offenbar weil der Entladungsstrom hier nicht oder nur schwach einwirken konnte.

Auf die angegebene Art gelingt es übrigens auch, auf eine dünne kapillare Blutschicht unter einem gewöhnlichen Deckglase den vollen

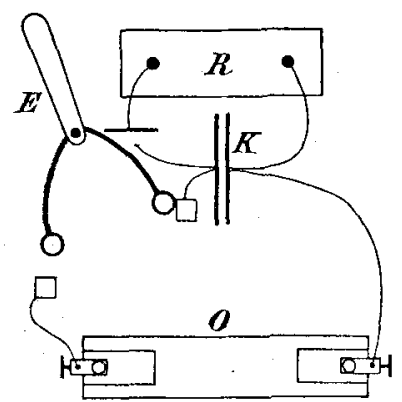

Fig. 2.

Entladungsstrom wirken zu lassen. Man bringt in die Mitte des $50 \mathrm{~mm}$ langen Raumes zwischen den Stanniolbelegen einen Tropfen Blut, and bedeckt ihn mit einem Deckglas von $15 \mathrm{~mm}$ Seite. Dann bedeckt man mittels eines Glasstabes den übrigen Raum zwischen Deckglas und Stanniol mit einer nicht zu dünnen Blutschicht. Lässt man jetzt in der angegebenen Weise eine einzige Entladung durch das Blut gehen, so wird das Blut unter dem Deckglase augenblicklich aufgehellt. Dies ist wohl die energischste Form des Roll et t'schen Phänomens. Aber die Hoffnuug, unter dem Mikroskop diese plötzliche Aufhellung zu verfolgen, erfülte sich doch nicht. Zwar ist es ein Leichtes, ein starkes Objektiv $(\boldsymbol{E})$ auf die Blutkörper einzustellen. Sowie man aber das Objekt zunächst einseitig mit dem Kondensator verbindet, findet eine so gewaltige Strömung unter dem Deckglase statt, dass jede Beobachtung unmöglich wird. Durch mannigfache Versuche habe ich mich überzeugt, dass diese Strömung mit der Ladungsrichtung des Kondensators nichts zu thun hat, sondern eine reine, vorläufig nicht bestimmter zu begründende, Kapillaritätserscheinung ist; die Adhäsionsverhältnisse zwischen Blut und Glas werden durch die elektrische Spannung offenbar wesentlich beeinflusst. Bei absichtlich durch Druck auf das Deckglas sehr dünn gemachter Blutschicht bleibt diese Bewegung aus; jetzt aber ist die Schicht nicht mehr genügend für die Entladung leitungsfähig, d. h. sie wird bei derselben von einem mächtigen Funken übersprungen.

Das Endresultat ist also, dass es zwar gelingt, dünne Blutschichten unter einem Deckglase aufzuhellen, und zwar augenblicklich, dureh eine einzige Entladung, dass es aber nicht gelingt, diesen Vor- 
gang bei hinreichender Vergrösserung direkt mikroskopiseh zu beobachten.

Die spezifische Wirkung der Entladungsschläge kann weder auf der Höhe der Potentialdifferenz noch auf der nur einsinnigen Wirkung beruhen. Denn bei Anordnung 1 und 2 ist die Potentialdifferenz zweifellos höher als bei 8 , und einsinnig sind die Ströme auch bei den Anordnungen 2, 4 und 6 . Ebensowenig kann es sich um die Energiemengen handeln, welche bei Anordnung 7 offenbar weit grösser sind als bei 8 . Offenbar also ist das, worauf es ankommt, nur der zeitliche Verlauf des Vorganges, oder genauer, die Elektrizitätsmenge, welche sich auf ein Zeittheilchen im Querschnitt der Flüssigkeit zusammendrängt, wofür man auch sagen kann die maximale momentane Stromdichte. Es muss aus den Versuchen gefolgert werden, dass, wenn diese auch nur in einem ungemein kleinen Zeitelement eine gewisse Grösse überschreitet, eine Bedingung für die Existenz der Blutkörper in ihrem normalen Status beseitigt wird, so dass dieselben rasch Bestandtheile an das Serum abgeben.

Dass diese letztere Zerstörung keine ganz unmittelbare Wirkung des Stromes ist, ergiebt sich schon aus Rollett's Beobachtung, dass jeder Funke eine Nachwirkung im Sinne der Aufhellung hat, und noch unmittelbarer aus den zuletzt angeführten Versuchen über die Wirkung einer einzigen Entladung in dünner Schicht. Hinsichtlich des vermittelnden Zwischengliedes ist als nächstliegende Möglichkeit die zu prüfen, ob die Entladung an den osmotischen Eigenschaften der Blutkörper etwas Wesentliches ändert.

Da mir Versuche am Blute selbst in dieser Richtung zunächst wenig Aussichten zu bieten schienen, wandte ich mich zu Versuchen über die Wirkung von Entladungsströmen auf einen leichter übersehbaren osmotischen Apparat, nämlich auf eine Pfeffer'sche Zelle. Die betr. Versuche sind grösstentheils schon vor mehr als Jabresfrist ausgeführt.

\section{Versuche an Niederschlagsmembranen.}

Der Apparat (es wurden in der Regel mehrere solche gleichzeitig in Thätigkeit gesetzt, weil die Herstellung ziemlich zeitraubend ist) war dem von. W. Pfeffer ${ }^{1}$ ) benutzten nachgebildet und wird durch

1) Osmotische Untersuchungen S. 1-14. Leipzig 1877. 
Fig. 3 veranschaulicht (in $2 / 5$ der natürlichen Grösse). Nachdem es mir nach manchen Fehlversuchen gelungen war, geeignete Thonzellen mir zu verschaffen, wurde in dieselben ein T-förmiges Glasrohr eingekittet $^{1}$ ), und genau nach Pfeffer's Vorschriften der Innenfläche des Thoncylinders eine Membran aus Ferrocyankupfer aufgelagert. Die obere und die seitliche Mündung wurden hierauf mit guten Gummistöpseln $S, S^{\prime}$ verschlossen. In dem seitlichen Stöpsel steckt ein enges geschlossenes Quecksilbermanometer $M$ mit Erweiterung im kurzen Schenkel. In dem

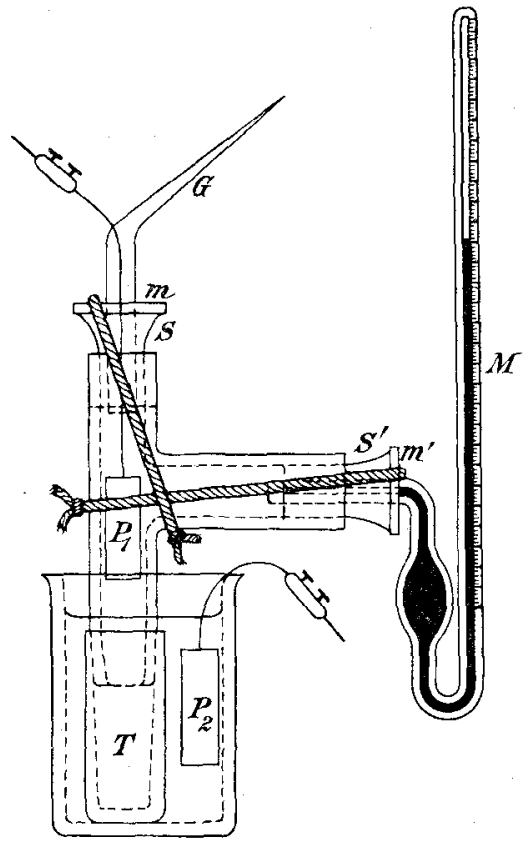

Fig. 3. oberen Stöpsel steckt ein schräg abgebogenes ausgezogenes Glasrohr $G$. Um das Gleiten der Gummistöpsel bei hohem Innendrucke zu verhindern, erwies es sich zweckmässig, die entsprechenden Ränder des $\mathrm{T}$-Rohres etwas nach innen aufzuwulsten, so dass sie den Gummistöpsel etwas würgen. Ferner sichere ich mich gegen das Herausgleiten durch einen sehr einfachen Verband: auf die ebene Endfäche der Stöpsel wird je eine runde Messingscheibe $m, m^{\prime}$ gelegt, durch deren centrale Bohrung das Ansatzrohr, resp. Manometerrohr, gerade hindurch geht. Die Scheibe wird hierauf durch einen sehr fest anziehbaren starken Bindfaden, welcher das T-Rohr umfasst, kräftig aufgepresst ${ }^{2}$ ). Durch das obere Glasrohr ist ein Platindraht, welcher seitlich eintritt und eingeschmolzen

1) Sorgfältige Einkittung mit einer bestimmten Siegellacksorte, welche mit sehr wenig Terpentinöl versetzt wurde, genügte für die höchsten, in meinen Versuchen vorkommenden Drucke (etwas über 3 Atmosphären), ohne dass das von Pfeffer empfohlene äussere, mit Bleiglättekitt gefüllte Schutzrohr nöthig war.

2) Ein für solche Aufgaben sehr geeigneter Ziehknoten ist von mir beschrieben und abgebildet in meinem Leitfaden für das physiologische Praktikum. Leipzig 1898. S. 148. 
ist, hindurchgeführt; an sein inneres Ende ist eine Platinplatte $P_{1}$ angeniethet. Die Auffüllung der Innenflussigkeit ( 3 procentige Ferrocyankalium-Lösung ${ }^{1}$ ) mit den unten zu erwähnenden Zusätzen) muss so geschehen, dass der Innenraum keine einzige Luftblase enthält und die Flüssigkeit in den ausgezogenen Theil des oberen Ansatzrohres hineinragt, welcher endlich abgeschmolzen wird.

Die Thonzelle ist bis über die Verkittung hinaus in 3 procentige Kupfersulphatlösung eingetaucht, welche sich in einem nicht zu grossen Gefässe befindet. In die Kupferlösung ist ebenfalls eine an einen Platindraht angeniethete Platinplatte $P_{2}$ eingetaucht.

Die zur Bildung der Niederschlagsmembran anfangs als Innenflüssigkeit benutzte 3 procentige Ferrocyankalium-Lösung wurde nach Bildung der Membran durch die Versuchsflüssigkeit ersetzt, welche aus derselben Lösung, jedoch versetzt mit 1,5\% eines anderen neutralen Salzes oder auch Rohrzucker, bestand. Meist wurde Kaliumnitrat als Zusatz gewählt; über andere Salze s. unten. Erst nach Füllung mit der Versuchslösung wurde der oben erörterte Verschluss hergestellt.

Der in den Versuchen nach 1-2 Tagen, zuweilen weit langsamer, erreichte am Manometer abgelesene osmotische Druck stieg auf $1 / 2$ bis über 3 Atmosphären, und nahm dann eine stationäre Höhe an. Sobald diese erreicht war, wurde mit dem eigentlichen Versuch begonnen, welcher in Durchleitung starker Funkenentladungen mittels der beiden beschriebenen Platinelektroden bestand.

$\mathrm{Zu}$ den Funkenentladungen diente die oben angeführte Anordnung 8 und alle oben beschriebenen Apparate. Die beiden Platinplatten des osmotischen Apparates wurden statt der Zinkelektroden des Troges $T$ (Fig. 1) mit dem Schlüssel $S$ verbunden. Der Erfolg sehr zahlreicher und hinsichtlich der Dauer und Stärke der Einwirkung mannigfach variirter Versuche ist folgender:

Schon sehr wenige Entladungen (etwa fünf in Sekundenintervall) genügen, um die Haupterscheinung hervorzubringen, nämlich ein beträchtliches Sinken des Manometers, welches wesentlich erst nach dem Schluss der Entladungsreihe eintritt und sich Stunden lang hinziehen kann. Oft sieht man bei jedem Funken ein leichtes Zucken des Quecksilbermeniskus im Manometer.

1) Es sei hier erwähnt, dass die Ferrocyankalium-Lösungen bei längerem Aufbewahren eine schon nach wenigen Tagen beginnende dunklere Gelbfärbung annehmen. Dieselbe rührt von langsamer Oxydation mit Bildung von etwas Ferricyankalium her. 
Um diese Erscheinung in geringem $\mathrm{M}$ a ass e hervorzurufen, genügt, namentlich bei sehr hohem osmotischem Druck und an frischen Membranen, schon die Durchleitung der Wechselströme eines gewöhnlichen, mit einem einzigen Element und W agner'schem Hammer betriebenen kleinen Induktoriums.

Lässt man eine längere Reihe starker Entladungen (etwa 60-100 und mehr) hindurchgehen, so sieht man während derselben ein Steigen des Manometers, welches aber gegen Ende wieder zurückzugehen beginnt, und sogar in Sinken gegen den ursprünglichen Stand übergehen kann. Nach Schluss der Entladungen tritt stets beträchtliches Sinken ein, welches sich viele Stunden fortsetzt. Am folgenden Tage findet man den Druck wieder gestiegen, und allmählich (in zehn Stunden oder mehr) erreicht derselbe wieder nahezu die ursprangliche Höhe, aber anscheinend niemals vollständig.

Wenige Versuchsbeispiele werden genügen, um von der Grössenordnung der Frscheinungen eine Vorstellung zu geben.

Beispiel 1 (Normalstand des Manometers $117 \mathrm{~mm}$ ). Die Zelle zeigte einen osmotischen Druck von uber drei Atmosphären, wurde am 19. Juni einem Versuche mit gewöhnlichen Induktionsströmen unterworfen, welcher den Druck zu starkem Sinken brachte, das aber am 20. Juni durch Steigen bis auf etwa zwei Atmosphären einigermassen wieder ausgeglichen war.

Entladungsversuch am 20. Juni. Stand des Manometere um $12^{\text {h }} 40^{\prime} 37$, entsprechend 2,16 Atmosphären. Von $12^{\text {h }} 40^{\prime}$ bis $12 \mathrm{~h} 42^{1 / \mathrm{a}^{\prime}}$ wird pro Sek. je etwa eine Entladung durcbgeleitet.

\begin{tabular}{|c|c|c|c|c|c|}
\hline \multirow{2}{*}{ 20. Juni 1901.} & \multirow{2}{*}{$\begin{array}{l}\text { Zeit } \\
\mathrm{h},\end{array}$} & \multirow{2}{*}{$\begin{array}{l}\text { Stand des } \\
\text { Mano- } \\
\text { meters } \\
\text { mm }\end{array}$} & \multirow{2}{*}{$\begin{array}{l}\text { Atmo- } \\
\text { sphären }\end{array}$} & \multicolumn{2}{|c|}{ Differenz in Atm. } \\
\hline & & & & im Ganzen & pro Min. \\
\hline \multirow[t]{2}{*}{$\begin{array}{l}\text { Während der Ent- } \\
\text { ladungen: }\end{array}$} & $\begin{array}{l}12.40 \\
12.41 \\
12.42^{1 / 2}\end{array}$ & $\begin{array}{l}37 \\
36,5 \\
43\end{array}$ & $\begin{array}{l}2,16 \\
3,21 \\
1,72\end{array}$ & - & $\overline{-}$ \\
\hline & $\begin{array}{l}12.46 \\
12.47^{1 / 2} \\
12.51^{1 / 2} \\
12.54 \\
1.2 \\
1.7 \\
1.20 \\
1.26 \\
4.36\end{array}$ & $\begin{array}{c}54 \\
60 \\
69 \\
73 \\
84 \\
89,5 \\
99 \\
101 \\
98,5\end{array}$ & $\begin{array}{l}1,16 \\
0,95 \\
0,70 \\
0,60 \\
0,39 \\
0,31 \\
0,18 \\
0,16 \\
0,25\end{array}$ & $\begin{array}{l}-1,00 \\
=0,21 \\
=0,25 \\
=0,10 \\
=0,21 \\
=0,08 \\
=0,13 \\
=0,02 \\
+0,11\end{array}$ & $\begin{array}{l}-0,167 \\
-0,14 \\
-0,06 \\
-0,04 \\
-0,026 \\
-0,016 \\
-0,010 \\
-0,003 \\
+0,009\end{array}$ \\
\hline $\begin{array}{l}\text { Nach zwei Tagen: } \\
\text { 22. Juni .... }\end{array}$ & 11.57 & 65 & 0,80 & - & - \\
\hline
\end{tabular}


Beispiel 2 (Normalstand des Manometers $151 \mathrm{~mm}$ ). 23. Juni. Stand $51,25=1,97$ Atmosphären. Entladungen 5 Minuten lang, zwei Funken pro Sek., Funkenlänge $5 \mathrm{~mm}$.

\begin{tabular}{|c|c|c|c|c|c|}
\hline \multirow{2}{*}{ 23. Juni 1901.} & \multirow{2}{*}{$\begin{array}{l}\text { Zeit } \\
\text { h , }\end{array}$} & \multirow{2}{*}{$\begin{array}{c}\text { Stand des } \\
\text { Mano- } \\
\text { meters } \\
\text { mm }\end{array}$} & \multirow{2}{*}{$\begin{array}{l}\text { Atmo- } \\
\text { sphären }\end{array}$} & \multicolumn{2}{|c|}{ Differenz in Atm. } \\
\hline & & & & absolut & pro Min. \\
\hline \multirow[t]{2}{*}{ Entladungen: } & $\begin{array}{l}10.0 \\
10.1 \\
10.2 \\
10.3 \\
10.4 \\
10.5\end{array}$ & $\begin{array}{l}51,25 \\
49 \\
47,5 \\
45,5 \\
45 \\
45,25\end{array}$ & $\begin{array}{l}1,97 \\
2,08 \\
2,18 \\
2,32 \\
2,36 \\
2,34\end{array}$ & $\begin{array}{l}-\overrightarrow{0} \\
+0,11 \\
+0,10 \\
+0,14 \\
+0,04 \\
-0,02\end{array}$ & $\begin{array}{r}-\overline{0} \\
+0,11 \\
+0,10 \\
+0,14 \\
+0,04 \\
-0,02\end{array}$ \\
\hline & $\begin{array}{l}10.7 \\
10.7^{1 / 2} \\
10.18 \\
10.25 \\
10.38 \\
11.13 \\
11.46 \\
12.31 \\
12.51 \\
3.48\end{array}$ & $\begin{array}{c}50 \\
52 \\
67 \\
74 \\
83 \\
95,5 \\
100 \\
101 \\
101,25 \\
99\end{array}$ & $\begin{array}{l}2,02 \\
1,90 \\
1,25 \\
1,04 \\
0,82 \\
0,58 \\
0,51 \\
0,50 \\
0,49 \\
0,525\end{array}$ & $\begin{array}{r}-0,32 \\
-0,08 \\
-0,65 \\
-0,19 \\
-0,22 \\
-0,24 \\
-0,07 \\
-0,01 \\
-0,01 \\
+0,035\end{array}$ & $\begin{array}{l}-0,16 \\
-0,16 \\
-0,06 \\
-0,027 \\
-0,017 \\
-0,007 \\
-0,002 \\
-0,0002 \\
-0,0005 \\
+0,0002\end{array}$ \\
\hline Nächster Tag: & 9.32 & 96 & 0,57 & - & - \\
\hline
\end{tabular}

Druck steigt langsam weiter.

Die letzte Kolumne der Tabellen ergiebt, dass das Sinken des Druckes mit abnehmender Geschwindigkeit erfolgt.

Für das während der Entladungen selbst erfolgende Steigen des Manometers ergiebt sich als nächstliegende Erklärung die Erwärmung in Folge der Durchströmungen, welche bei nicht allzu kurzen Entladungsreihen obne Weiteres von aussen mit den Fingern fühlbar ist. Die geschlossene Zelle mit ihrem Manometer stellt eine Art Thermometer mit sehr gtossem Gefäss dar, und schon mässige Erwärmungen müssen die Quecksilbersäule zu beträchtlichem Steigen bringen, da ein Austritt von Wasser durch die halbdurchgängige Membran nur sehr langsam erfolgen kann. Es hat daher auch wenig Bedeutung, dieses Ansteigen des Manometers in Drucksteigerung umzurechnen. Dass diese Erklärung reichlich genügt, ergiebt folgende Erwägung, , welche einen weiteren Schluss zulässt. Der Rauminhalt der Zellen mit dem $\mathrm{T}$-Rohr beträgt ungefähr 
$25 \mathrm{~cm}^{3}$ (die einzelnen Apparate sind natürlich in dieser Richtung verschieden), der Querschnitt der Manometerröhren im Lichten etwa $0,5 \mathrm{~mm}^{2}$. Das Steigen des Quecksilbers betrug in Beispiel 2 etwa $6 \mathrm{~mm}$, also die Volumzunahme der Innenflusssigkeit etwa $3 \mathrm{~mm}^{3}$ oder etwa $3 / 25000=$ etwas unter $1 / 8000$ des Gesammtvolums. Zu dieser kubischen Ausdehnung würde schon eine Erwärmung um etwa ${ }^{1 / 2}-1^{0}$ genügen; die wirkliche von aussen fühlhare Erwärmung ist aber offenbar beträchtlich grösser. Man kann hieraus schliessen, dass entweder die thermische Ausdehnung wirklich Wasser durch die Niederschlagsmembran auspresst, oder dass der zweite, erst gegen Schluss der Entladungen oder nach denselben auftretende Prozess, die Druckabnahme, schon während der Entladungen, vielleicht schon von Anfang an, eine Rolle spielt. Diese Umstände erklären auch zur Genüge, warum die anfängliche Drucksteigerung in den verschiedenen Versuchen in sehr wechselndem Grade zur Erscheinung kommt.

Die zweite Erscheinung, die Drucksenkung, hat zweifellos ihren Hauptablauf erst nach Beendigung der Entladungen. Hiermit fallen sofort alle Erklärungsversuche, welche etwa an direkte Stromwirkungen, z. B. Elektrotransfusion (elektrische Osmose), anschliessen. Fine solche Wirkung ist allerdings a priori nicht ausgeschlossen, da die oszillatorischen Entladungen des Kondensators eine bestimmte Hauptrichtung haben, also wie auf Gleichströme superponirte Wechselströme wirken werden. Spezieller wird aber die Erklärung aus elektrischer Osmose durch die Thatsache völlig ausgeschlossen, welche ich oft festgestellt habe, dass ein Polwechsel zwischen Kondensator und Platinelektroden auf die Erscheinung keinerlei Einfluss hat.

Auf eine wirkliche Erklärung leitete mich die zuerst zufällig gemachte und dann stets bestätigte Beobachtung, dass das gläserne $T$-Rohr an seiner Innenffäche längere Zeit nach den Entladungen einen ungemein zarten rothen Anflug zeigt, welcher nun bestehen bleibt. Man erkennt ihn auch an dem oberen Ansatzrohr und am deutlichsten im $T$-Rohr, wenn man die Gummistöpsel herausnimmt; die mit ihnen in Berührung gewesene Glasfläche sticht durch ihre Farblosigkeit von der schwach röthlichen Nachbarschaft stark ab. Dieser Anflug, welcher in Säuren unverändert bleibt, in Alkalien aber sofort verschwindet, besteht offenbar aus Ferrocyankupfer. Die Niederschlagsmembran ist also durch die hindurchgehenden Entladungen angegriffen worden. Entweder 
hat sich, was ich für weniger wahrscheinlich halte, durch die blosse Erwärmung, welche unmittelbar an der Membran besonders stark sein mag, etwas Ferrocyankupfer in der darüber stehenden Ferrocyankalium-Lösung gelöst und zunächst unter dem Einfluss der Ströme gleichmässig in der ganzen Flüssigkeit vertheilt, oder es hat in gewissem Grade eine mechanische Absplitterung von äusserst feinen Partikeln des Niederschlages und Vertheilung derselben in der Lösung stattgefunden. Dass solche Partikelchen nicht auch in die Aussenflüssigkeit gelangen können, erscheint leicht begreiflich, da dieselben hierzu erst eine dicke Thonsehicht passiren müssten, in welcher sie durch die Enge und Oberflächenwirkung der Poren zurückgehalten werden müssen. Die in der Innenflüssigkeit entweder gelösten oder suspendirten Partikel werden sich nach Eintritt von Abkühlung und Ruhe abscheiden müssen, und zwar durch oberflächenwirkung vorzugsweise an der Glaswand, zumal sie zu fein sind, um durch ihre Schwere in die Thonzelle hinabzusinken.

Wie aber die zweifellos vorhandene Schwächung oder sonstige Schädigung der Niederschlagsmembran zu der Drucksenkung fübrt, ist eine noch zu erledigende Frage. Zunächst könnte man daran denken, dass die Entladungsschläge unabhängig von der Membranveränderung den osmotischen Druck direkt herabsetzen; hierzu müsste aber die Anzahl der Moleküle oder Ionen in der oberen Lösung irgendwie abnehmen, also der Dissoziationszustand sich vermindern. Für eine solche Annahme liegt weder irgend eine Thatsache vor, noch ist es denkbar, dass eine derartige Veränderung die Entladungen selbst irgendwie überdauert, was man annehmen müsste, um die Erscheinungen zu begreifen.

Schon mehr Wảhrscheinlichkeit würde die Möglichkeit haben, dass der Angriff auf die Membran deren charakteristische Eigenschaft, nur Wasser und nicht andere Moleküle oder Ionen hindurchzulassen, für längere Zeit unvollkommen macht oder aufhebt; die Zelle würde hierdurch sofort ungeeignet werden, den wahren osmotischen Druck der Innenlösung am Manometer anzugeben; ausserdem würde auch durch den Austritt gelöster Bestandtheile dieser Druck wirklich abnehmen. Wenn diese Erklärung richtig wäre, müsste anscheinend der neben dem Ferrocyankalium in der Innenflüssigkeit gelöste Stoff nach beendigtem Sinken des Druckes in der äusseren Kupferlösung nachweisbar sein. 
Um dies zu entscheiden, habe ich in den Versuchen mit Kaliumnitrat wiederholt die Kupferlösung auf Salpetersäure untersucht ${ }^{1}$ ), aber nie eine Spur derselben gefunden. Ebensowenig gelang es in Versuchen, in welchen dem Ferrocyankalium statt des Kaliumnitrats Kaliumjodid, Kaliumrhodanid, Rohrzucker zugesetzt war, jemals in der Aussenflüssigkeit die entsprechenden Bestandtheile nachzuweisen. Die Membran hatte also ibre halbdurchlässige Beschaffenheit scheinbar nicht eingebusst.

Eine andere zu erwägende Möglichkeit bestand darin, dass vielleicht der Widerstand der Membran gegen den Wasserdurchtritt vermindert wird. Man könnte einen Augenblick daran denken, dass in Folge dessen der hohe Druck in der Zelle Wasser nach aussen durchpresst. Aber dieser Gedankengang wäre fehlerhaft. Der osmotische Ueberdruck in der Zelle kann sich ja nur dadurch am Manometer zu erkennen geben, dass das Volum der Innenflüssigkeit zuzunehmen im Stande ist, d. h. durch Eintritt von Wasser durch die Membran. Mit anderen Worten: der osmotische Ueberdruck saugt so lange Wasser durch die Membran ein ${ }^{2}$ ), bis der entstandene Gegendruck einer Quecksilbersäule oder komprimirter Luft dem Ueberdruck gleich geworden ist. Also kann erleichterter Wasserdurchtritt den Gleichgewichtszustand, wenn derselbe einmal da ist, nicht beeinflussen, und wäre er noch nicht erreicht, so würde erleichterter Wasserdurchtritt im Gegentheil seine Herstellung beschleunigen, d. h. das Steigen des Quecksilbers auf die entsprechende Höhe schneller herbeiführen, nie aber dasselbe zum Sinken bringen.

Trotzdem habe ich direktere Versuche darüber angestellt, ob etwa die Veränderung der Niederschlagsmembran durch die Entladungen deu Durchtritt von Wasser erleichtert. Eine wie sonst hergestellte Thonzelle mit innen aufgelagerter Niederschlagsmembran von Ferrocyankupfer wurde innen und aussen mit ders elb en Flüssig-

1) Die ganze Kupferlösung wurde mit reinem Kaliumhydrat ausgefällt und dann mit dem Niederschlag bis zu völliger Schwärzung desselben gekocht, das Filtrat mit reiner Schwefelsäure übersättigt und in bekannter Weise auf Salpetersäure geprüft.

2) Die einfachste Vorstellung für diese bekanntlich auf den ersten Blick paradoxe Thatsache dürfte die sein, dass der osmotische Druck nur auf die eigentliche Wandsubstanz zu wirken vermag und nicht auf die Poren, auf welche sich das Expansionsbestreben der in sie nicht eindringenden Ionen nicht erstreckt. 
keit in Berührung gebracht, und zwar mit destillirtem Wasser, Leitungswasser oder 3 procentiger Kupfersulphat-Lösung. Der Seitenarm des $T$-Rohres war, statt mit einem geschlossenen Manometer, mit einem $1^{1 / 2} \mathrm{~m}$ langen vertikalen Glasrohr in Verbindung, das zwischen Gummistöpsel und dem hohen, vertikalen, offenen Schenkel nach unten U-förmig ausbog und in dem an das $T$-Rohr grenzenden Schenkel eine kugelige Erweiterung hatte, ferner oben einen kleinen Eingusstrichter trug. Der obere Verschluss des $T$-Rohres war wie sonst und liess auch hier den Platindraht der inneren Elektrode durch; die andere Elektrode befand sich wiederum in der Aussenflüssigkeit. Füllt man das vertikale Druckrohr auf eine Höhe von etwa 115 bis $125 \mathrm{~cm}$ mit Quecksilber, so muss natürlich dieser Druck allmählich Wasser durch die Zelle nach aussen treiben, und ein definitives Gleichgewicht wird erst nach sehr langer Zeit erreicht. Bei destillirtem Wasser würde dasselbe erst nach völliger Ausgleichung des Ueberdrucks eintreten. Bei einer Kupfersulphat-Lösung würde durch den Austritt von destillirtem Wasser alsbald eine Koncentrationsdifferenz zwischen Innen- und Aussenflüssigkeit und dadurch ein zunehmender osmotischer Ueberdruck der ersteren herbeigeführt werden und das Quecksilber zum Stillstand kommen, sobald sein Druck diesem Ueberdruck gleich geworden ist.

In der That sieht man das Quecksilberniveau, welches auch die Flüssigkeit sein mag, langsam herabgehen; das Sinken beträgt pro Tag nur etwa $10-20 \mathrm{~mm}$, so dass man sehr wohl Entladungsversuche anstellen kann.

Diese ergeben, wie in den früheren Versuchen, während der Entladung ein geringes Steigen des Niveau's, welches dann wieder zu sinken beginnt; dabei wird das Glas deutlich warm, und es zeigt sich wiederum nachher der zarte rothe Anflug. Nach Aufhören der Entladungen setzt sich das Sinken energischer fort, auch hier mit abnehmender Geschwindigkeit, und lässt sich beliebig lange verfolgen. Ein Wiederansteigen findet nicht statt. Offenbar geht das durch die Entladungen bewirkte Sinken schliesslich in das langsame auch ohne jene vorhandene über.

Das Steigen des Niveau's betrug gewöhnlich nur wenige (höchstens 3-4) Millimeter, das Sinken, dessen Ende eben nicht bestimmbar ist, weil kein Gleichgewicht oder Wiederansteigen eintritt, meist gegen $40-50$, in einigen Versuchen bis gegen $400 \mathrm{~mm}$. Würde 
man diese Beträge in Atmosphären umrechnen und mit den Druckänderungen der eigentlichen osmotischen Versuche vergleichen, so würden sie äusserst gering erscheinen; das Steigen beträgt nur 0,004-0,005, das Sinken nur 0,05-0,06, höchstens 0,5 Atm. Dagegen sind die durchgetretenen Wasservolumina beträchtlich, nämlich, da das Quecksilberrohr hier ca. $12 \mathrm{~mm}^{2}$ inneren Querschnitt hatte, bedeutet das Sinken um $50 \mathrm{~mm}$ einen Flüssigkeitsaustritt von $600 \mathrm{~mm}^{3}$, während die Versuche mit engem geschlossenem Manometer bei einer Drucksenkung von 3 auf 2 Atmosphären nur mit einem Austritt von etwa $5 \mathrm{~mm}^{3}$ Flüssigkeit verbunden waren. Um das Steigen auch hier durch thermische Ausdehnung zu erklären, müsste, da dasselbe kubisch etwa ${ }^{1 / 700}$ bis ${ }^{1 / 500}$ des Gesammtvolums ausmacht, eine Erwärmung um mehr als $15^{\circ}$ angenommen werden, was nicht das mindeste Bedenken hat.

Es war nun zu untersuchen, ob die ausgetretene Flüssigkeit blosses Wasser oder Kupfersulphat-Lösung war. Zu diesem Behufe bestimmte ich den Kupfergehalt, welcher ursprünglich in der Innenund Aussenflüssigkeit gleich war, in je $10 \mathrm{ccm}$ beider Flüssigkeiten durch Titriren mit einer Ferrocyankalium-Lösung, wobei als Endreaktion das Verhalten der Mischung gegen Eisenchlorid benutzt wurde. Während des Versuchs selbst und während der folgenden Stunden war das Verdunsten von Wasser aus der Aussenflüssigkeit durch Aufstellung dès Apparates in einer feuchten Kammer, aus welcher das Vertikalrohr herausragte, verhindert worden. Es ergab sich, dass zwar stets die Aussenflüssigkeit kupferärmer war als die Innenflüssigkeit, aber nicht in dem Grade, wie es hätte sein müssen, wenn $n$ ur Wasser ausgetreten wäre.

Man muss also den Schluss ziehen, dass die Entladungen die Niederschlagsmembran so verändern, dass sie für Wasser unter gleichem Druck leichter als vorher durchgängig wird, aber auch das gelöste Salz, wenn auch in geringerem Verhältniss als Wasser, durchtreten lässt.

Dieser letztere Schluss steht auf den ersten Blick in direktem Widerspruch mit dem oben S. 182 gezogenen, dass die Membran ihre halbdurchlässige Beschaffenheit durch die Entladungen seheinbar nicht einbüsse. Bei näherer Erwägung zeigt sich aber, dass Letzteres nicht völlig sicher ist. Nimmt man nämlich an, dass in den Hauptversuchen die Schädigung der Membran wirklich etwas 
von den Salzen der Innenflüssigkeit zum Austreten bringt, so folgt daraus noch nicht, dass dieselben in der Aussenflüssigkeit nachweisbar sein müssen. Denn jedes Quantum Ferrocyankalium, welches etwa in Gestalt äusserst feiner Lösungsfäden durch die Membran in die Thonzelle eindringt, muss sich sofort mit nener Niederschlagsmembran umhüllen. Nun ist aber das in Folge der Entladungen ausgetretene Volum äusserst gering. Nehmen wir den Manometerquersehnitt zu $0,5 \mathrm{~mm}^{2}$ an (diese Grösse hatte er, genauer 0,507 , beim weitesten der verwendeten Manometer), so betrug in Beispiel 1 das ausgetretene Volum (37 bis $101 \mathrm{~mm}$ Meniskusstand) $64 \cdot 0,5=$ $32 \mathrm{~mm}^{3}$, in Beispiel $2(51,25$ bis 101,25$) 50 \cdot 0,5=25 \mathrm{~mm}^{3}$. Diese Volumina sind so gering, dass sie wohl kaum aus der Substanz der Thonwand herauskamen. Das Volum der Wandsubstanz betrug für den nicht von Kitt bedeckten Theil des Thoncylinders über $2000 \mathrm{~mm}^{3}$. Es ist dabei zu erwägen, dass der Austritt wesentlich erst $\mathrm{nach}$ den Entladungen sich vollzieht und die während desselben sich bildenden neuen Niederschlagsmembranen also keinem Angriff unterliegen, folglich nicht $\mathrm{zu}$ erwarten ist, dass nachweisbare Mengen des nicht niederschlagbildenden Bestandtheils (Kaliumnitrat etc.) in die Aussenflüssigkeit gelangen können, zumal die austretende Lösung nach den letztangeführten Versuchen verdünnter ist als die Innenflüssigkeit.

Somit erklärt sich alles Beobachtete auf folgende Weise: Die Entladungen bewirken zunächst durch Erwärmung Ausdehnung der Innenflüssigkeit und Steigen des Manometers; ferner greifen sie die Niedersehlagsmembran, sei es durch partielle Auflösung; sei es durch mechanische Zerstäubung, derartig an, dass sie nicht allein für Wasser durchgängiger wird, sondern auch den gelösten Stoffen Durchtritt gestattet, und zwar für längere Zeit, bis die Membran sich wieder genügend regenerirt hat. In Folge dessen treibt der hohe Innendruck verdünnte Innenlösung durch die Membran heraus und sinkt schon während der Entladungen, noch mehr nach denselben beträchtlich. Die austretenden Quanta umgeben sich sofort mit neuer Niederschlagsmembran und sind an Volum wegen des engen geschlossenen Manometers so gering, dass sie grösstentheils oder ganz in der Thonwand bleiben; das beigemisehte Salz wird durch die neuen Niederschlagsmembranen verhindert, in nachweisbarer Menge in die Aussenflüssigkeit zu gelangen. 
Schliesslich will ich noch einige Erfahrungen mittheilen, welche ich im Laufe eines Jahres bei zahlreichen Wiederholungen des Hauptversuchs gemacht habe. Zeigt die Zelle nur geringen osmotischen Druck, so ist die Wirkung der Entladungen in der Regel unbedeutend. Da nun, wie die oben mitgetheilten Beispiele zeigen, das Sinken des Druckes sich noch beträchtlich fortsetzt, wenn schon ein sehr niedriges Niveau erreicht ist, so muss man anscheinend schliessen, dass eine unter hohem Druck stehende Niederschlagsmembran durch die Entladungsscbläge stärker angegriffen wird, als bei niedrigem Druck. Ferner zeigen sehr alte Niederschlagsmembranen oft nach den Entladungsschlägen nur unbedeutende Drucksenkung, und auch nicht den rothen Anflug, der oben erwähnt ist, sind also anscheinend weniger leicht angreifbar als frisch gebildete, und zeigen endlich keine Beeinflussung durch blosse Induktionsströme, selbst die des grossen Induktors (vgl. oben S. 178). Am stärksten ist Anflug und Drucksenkung bei solchen Zellen, welche in wenigen Tagen nach der Ingangsetzung einen hohen osmotischen Druck angenommen baben, was bei manchen Thonzellen picht zu erreichen ist. Am nächsten liegt wohl die Vermuthung, dass die Niederschlagsmembranen sich mit der Zeit verdicken und dann der angreifenden und zerstäubenden Wirkung der Entladungen mehr Widerstand entgegensetzen.

Die hier mitgetheilten Versuche mit Niederschlagsmembranen haben zunächst rein physikalisches Interesse. Möglicherweise aber liefern sie auch einen Beitrag zur Aufklärung des Rollettschen Phänomens. Freilich kann ihre Verwendung in dieser Richtung vorläufig nur unter bedeutendem Vorbehalt erfolgen. Allerdings verhalten sich nach neueren Untersuchungen ${ }^{1}$ ) die rothen Blutkörperchen in osmotischer Beziehung genau so, als wenn sie von einer nur für Wasser durchlässigen Membran umschlossen wären. Aber man kann doch eine wirkliche Membran dieser Art unmöglich annehmen, und welche Oberflächeneigenschaften die in Rede stehenden Erscheinungen bedingen, ist noch völlig in Dunkel gehüllt. Also wäre es vermessen, die von mir beobachtete Erscheinung ohne Weiteres auf die Verhältnisse der Blutkörper zu übertragen. Höchstens

1) Vgl. namentlich H. Koeppe, Physikalische Chemie in der Medicin. S. $33 \mathrm{ff}$. Wien 1900. 
kann man so weit gehen, den Entladungsströmen, d. h. (s. oben S. 175) enormen momentanen Stromdichten, eine angreifende Wirkung auf die Oberiäche der Blutkörper vermuthungsweise zuzuschreiben. Auch kann man sogar in Zweifel ziehen, ob überhaupt die Wand der Blutkörper von den Entladungsströmen in wesentlichem Grade durchsetzt wird. Wenn, wie manche Autoren angeben, die Blutkörper an dem galvanischen Leitungsvermögen des Blutes gar nicht betheiligt wären, könnte von einer solchen Durchsetzung natürlich keine Rede sein. In solcher Strenge kann aber jener Satz nicht gelten, und schon Rollett hat darauf hingewiesen, dass möglicherweise die Aufhellung damit zusammenhängt, dass das Innere der Blutkörper der Einwirkung der Entladungsschläge zugänglich ist, während es von anderen Strömen nur unbedeutende Zweige erhält. Anstatt aber mit Rollett ${ }^{1}$ ) anzunehmen, dass die Blutkörperchen gegen andere Ströme Isolatoren sind, scheint es mir einfacher und keiner Thatsache widersprechend, keinen so prinzipiellen Unterschied zwischen dem Inhalt der Blutkörper und ihrer Umgebung zu machen. Es genügt ja vollständig die Annahme, dass jener Inhalt sehr viel schlechter als das Serum leitet und daher nur geringe Antheile der Ströme die Trennungsfläche durchsetzen. Dann werden auch bei Entladungsströmen entsprechende Antheile durch die Blutkörper gehen, und man braucht nicht die sonst kaum zu begründende Annahme zu machen, dass Entladungsströme sich in Bezug auf ihren Verlauf anders als andere Ströme verhalten.

Ein weiterer Umstand, welcher bedenklich macht, aber nicht entscheidend ist, besteht darin, dass die Wirkung auf Niederschlagsmembranen oft schon bei blossen Induktionsströmen in gewissem Grade eintritt, während, wie wir gesehen haben, diese auf Blut ohne jede Wirkung sind, sobald Erwärmung ausgeschlossen ist.

In Erwägung könnte noch gezogen werden, dass nicht leitende suspendirte Partikelchen bekanntlich unter dem Einfluss von Strömen Aufreihungen zeigen (W eyl), welche auf eine dielektrische Polarisation deuten. Man könnte vielleicht annehmen, dass dies der Modus ist, welcher $\mathrm{zu}$ einem Angriff auf die Blutkörperchen führt, da die analoge Erscheinung von mir auch an diesen beobachtet ist $^{2}$ ). Es

1) A. a. O. S. 249 unten.

2) Vgl. meine frühere Arbeit S. 166. 
188 L. Hermann: Versuche über die Wirkung von Entladungsschlägen etc.

wäre nicht undenkbar, dass, wenn diese Polarisation so gewaltig und plötzlich ist wie bei Entladungsströmen, sie einen Angriff auf die Körperchen ausübt.

Uebrigens ist auch die hier mitgetheilte Wirkung der Entladungsströme auf Pfeffer'sche Zellen, resp. deren Niederschlagsmembran, zunächst physikalisch noch nicht erklärbar. Ich habe aber nach brieflicher Mittheilung die Hoffnung, dass sie durch weitere Untersuchungen von fachmännischer Seite ihrer Aufklärung sich nähern wird. 